\title{
HEEGAARD FLOER HOMOLOGY OF MAPPING TORI II
}

\author{
STANISLAV JABUKA, THOMAS MARK
}

\begin{abstract}
We extend the techniques in a previous paper to calculate the Heegaard Floer homology groups $\mathrm{HF}^{+}(M, \mathfrak{s})$ for fibered 3-manifolds $M$ whose monodromy is a power of a Dehn twist about a genus- 1 separating circle on a surface of genus $g \geq 2$, where $\mathfrak{s}$ is a non-torsion $\operatorname{spin}^{c}$ structure on $M$.
\end{abstract}

\section{INTRODUCTION}

After their introduction by Ozsváth and Szabó in a remarkable series of papers [4] 5. 6] 7], Heegaard Floer homology groups and related invariants of 3- and 4dimensional manifolds have rapidly become central tools in low-dimensional topology. This article is concerned with the calculation of the Heegaard Floer groups associated to certain fibered 3-manifolds, and can be seen as a continuation of, or supplement to, the work in 2]. In the latter paper, the authors considered 3manifolds $Y$ fibered over $S^{1}$ with fiber a surface $\Sigma_{g}$ of genus $g>1$ and monodromy given as certain combinations of Dehn twists along circles in $\Sigma_{g}$. Specifically, let $\gamma$ and $\delta$ denote a pair of nonseparating circles on $\Sigma_{g}$ that intersect transversely in a single point, and let $\sigma$ denote a circle that separates $\Sigma_{g}$ into components of genus 1 and $g-1$. Letting $t_{\gamma}, t_{\delta}$, and $t_{\sigma}$ denote the right-handed Dehn twists about $\gamma, \delta$, and $\sigma$, the monodromies considered in [2] were those of the form $t_{\gamma}^{n} t_{\delta}^{m}$ for $n, m \in \mathbb{Z}$ and $t_{\sigma}^{ \pm 1}$. For technical reasons the calculations of that paper did not apply to the case of other powers of the separating twist; part of the purpose of this paper is to make the necessary extensions in the arguments so as to include those cases.

Seen in a broader context, the present work extends to more general circumstances a result initially used in [8] and reformulated and applied in 22. Namely, given a nullhomologous knot $K$ in a closed oriented 3-manifold $Y$ we determine (under some hypotheses) the Heegaard Floer homology groups $\mathrm{HF}^{+}\left(Y_{0}, \mathfrak{s}\right)$ of the result $Y_{0}$ of 0 -framed surgery along $K$ in a $\operatorname{spin}^{c}$ structure $\mathfrak{s}$ whose first Chern class is not torsion, in terms of the "knot complex" $C F K^{\infty}(Y, K)$. This result is stated formally in Theorem 4 below, and the calculation of the Heegaard Floer groups for the fibered 3-manifolds mentioned above is given as an application.

For an orientation-preserving diffeomorphism $\phi$ of a surface $\Sigma_{g}$, we denote by $M(\phi)$ the mapping torus $\Sigma_{g} \times[0,1] /(x, 1) \sim(\phi(x), 0)$. For $\sigma$ a genus-1 separating circle on $\Sigma_{g}$ as above, note that $M\left(t_{\sigma}^{n}\right)$ has the homology of $\Sigma_{g} \times S^{1}$ for any $n \in \mathbb{Z}$. It follows that the following two conditions uniquely determine a spin ${ }^{c}$ structure $\mathfrak{s}_{k}$ on $\Sigma_{g}$ :

(i) $\left\langle c_{1}\left(\mathfrak{s}_{k}\right),[c] \times S^{1}\right\rangle=0$ for any class $[c] \in H_{1}\left(\Sigma_{g} ; \mathbb{Z}\right)$.

(ii) $\left\langle c_{1}\left(\mathfrak{s}_{k}\right),\left[\Sigma_{g}\right]\right\rangle=2 k$

According to the adjunction inequality for Heegaard Floer homology [] $H F^{+}\left(M\left(t_{\sigma}^{n}\right), \mathfrak{s}\right)=$ 0 unless $\mathfrak{s}$ satisfies (i) above, and also satisfies (ii) with $|k| \leq g-1$. We calculate here the Heegaard Floer groups in all remaining cases except $k=0$. 
To state the result, let $X(g, d)$ denote the graded group whose summand in degree $j$ is $H^{g-j}\left(\operatorname{Sym}^{d} \Sigma_{g} ; \mathbb{Z}\right)$. For a group $G$, we let $G_{(j)}$ denote the graded group isomorphic to $G$ with grading concentrated in degree $j$, and if $H$ is a graded group let $H[j]$ denote $H$ with the grading shifted by $j$.

The following is proved in sections 3.3 and 3.4 and incorporates Theorem 1.3 of [2] as the cases $n= \pm 1$.

Theorem 1. For $n \neq 0$, let $M\left(t_{\sigma}^{n}\right)$ denote the mapping torus of the $n$-th power of the right-handed Dehn twist around a genus 1 separating curve on a surface $\Sigma_{g}$ $(g \geq 2)$. Let $\mathfrak{s}$ be a nontorsion spin ${ }^{c}$ structure on $M\left(t_{\sigma}^{n}\right)$. Then the Heegaard Floer homology $\mathrm{HF}^{+}\left(M\left(t_{\sigma}^{n}\right), \mathfrak{s}\right)$ is trivial unless $\mathfrak{s}$ satisfies (i) and (ii) above. If these conditions hold we have an isomorphism of relatively graded groups

$$
\begin{aligned}
H F^{+}\left(M\left(t_{\sigma}^{n}\right), \mathfrak{s}\right)= & X(g-1, d-1) \otimes H^{*}\left(S^{1} \sqcup S^{1}\right)[\varepsilon(n)] \oplus \Lambda_{(g-d)}^{2 g-2-d} H^{1}\left(\Sigma_{g-1}\right) \\
& \oplus \bigoplus_{p=1}^{d}\left[\Lambda_{(g-d-p+1)}^{2 g-2-d+p} H^{1}\left(\Sigma_{g-1}\right)[\varepsilon(n)] \otimes H_{*}\left(\coprod_{|n|-1} S^{2 p-1}\right)\right],
\end{aligned}
$$

where $d=g-1-|k|$, and where $\varepsilon(n)=0$ if $n>0$ and $\varepsilon(n)=-1$ if $n<0$.

Note that while the Heegaard Floer groups admit further algebraic structure, in particular an action by the polynomial ring $\mathbb{Z}[U]$, the methods in this paper do not give information about that action: the isomorphism above is of $\mathbb{Z}$-modules only.

The following should be compared with results of Seidel [10] and Eftekhary [1] on the symplectic Floer homology of surface diffeomorphisms.

Corollary 2. There is an isomorphism of relatively graded groups

$$
H F^{+}\left(M\left(t_{\sigma}^{n}\right), \mathfrak{s}_{g-2}\right)= \begin{cases}H^{*}\left(\Sigma_{g}, C\right) & n>0 \\ H^{*}\left(\Sigma_{g} \backslash C\right) & n<0\end{cases}
$$

where $C$ denotes the union of $|n|$ pairwise disjoint pushoffs of the separating circle $\sigma \subset \Sigma_{g}$, and the right-hand side above denotes singular cohomology with coefficients in $\mathbb{Z}$.

Proof. We consider the case $n>0$. In the statement we take $k=g-2$, so that $d=1$. Since $X(g-1,0)=\mathbb{Z}_{(g-1)}$, the first line of (1) becomes $\mathbb{Z}_{(g-1)} \otimes\left(\mathbb{Z}_{(0)}^{2} \oplus\right.$ $\left.\mathbb{Z}_{(1)}^{2}\right) \oplus \Lambda^{2 g-3} H^{1}\left(\Sigma_{g-1}\right)_{(g-1)}=\mathbb{Z}_{(g-1)}^{2} \oplus \mathbb{Z}_{(g)}^{2} \oplus \mathbb{Z}_{(g-1)}^{2 g-2}$. The second line of (1) collapses to $\mathbb{Z}_{(g-1)} \otimes\left(\mathbb{Z}_{(0)}^{n-1} \oplus \mathbb{Z}_{(1)}^{n-1}\right)=\mathbb{Z}_{(g-1)}^{n-1} \oplus \mathbb{Z}_{(g)}^{n-1}$. Thus

$$
H F^{+}\left(M\left(t_{\sigma}^{n}\right), \mathfrak{s}_{g-2}\right) \cong \mathbb{Z}_{(g)}^{n+1} \oplus \mathbb{Z}_{(g-1)}^{2 g+n-1} .
$$

On the other hand, since $\Sigma_{g} \backslash C$ is the disjoint union of a punctured torus, a punctured surface of genus $g-1$, and $n-1$ annuli, we have

$$
H^{*}\left(\Sigma_{g}, C\right) \cong \mathbb{Z}_{(2)}^{n+1} \oplus \mathbb{Z}_{(1)}^{2 g+n-1}
$$

which agrees with the above modulo a shift in grading. The case $n<0$ follows similarly.

In the next section we state and prove Theorem 4 on the Floer homology of a manifold obtained by 0 -framed surgery on a nullhomologous knot; in Section 3 we prove Theorem 1 


\section{Floer Homology of 0-Surgeries}

The method we will use to make our calculation is an adaptation to slightly more general circumstances of the one used by Ozsváth and Szabó in [8] to determine the Heegaard Floer homology of the mapping torus of a single Dehn twist about a nonseparating curve on a surface of genus $g \geq 2$, in nontorsion $\operatorname{spin}^{c}$ structures.

Let $K \subset Y$ be a nullhomologous knot in a closed oriented 3-manifold $Y$, and let $Y_{n}(K)$ denote the result of $n$-framed surgery along $K$. Fix a spin ${ }^{c}$ structure $\mathfrak{s}$ on $Y$, and for integers $n>0$ and $k \in\{0, \ldots, n-1\}$ let $H F^{+}\left(Y_{n}(K), k\right)$ denote the Heegaard Floer homology of the surgered manifold in the $\operatorname{spin}^{c}$ structure $\mathfrak{s}_{k}$ defined as follows. Integer surgery corresponds to a cobordism $W_{n}$ from $Y$ to $Y_{n}(K)$, comprising a single 2-handle addition. Fixing a Seifert surface $F$ for $K$ we obtain a closed surface $\hat{F}$ in $W_{n}$ by capping off $F$ using the core of the 2handle . Now let $\mathfrak{s}_{k} \in \operatorname{Spin}^{c}\left(Y_{n}(K)\right)$ be the $\operatorname{spin}^{c}$ structure cobordant to $\mathfrak{s}$ by a $\operatorname{spin}^{c}$ structure $\mathfrak{r}$ on $W_{n}$ having

$$
\left\langle c_{1}(\mathfrak{r}),[\hat{F}]\right\rangle=n-2 k .
$$

Note that if, as will always be the case here, the $\operatorname{spin}^{c}$ structure on $Y$ is torsion and $H_{2}(Y ; \mathbb{Z})$ is torsion-free, then $\mathfrak{s}_{k}$ is independent of the choice of $F$.

According to [5], there is an integer surgeries long exact sequence

$$
\cdots \rightarrow H F^{+}\left(Y_{0}(K),[k]\right) \rightarrow H F^{+}\left(Y_{n}(K), k\right) \rightarrow H F^{+}(Y) \rightarrow \cdots
$$

Here the first term denotes the sum of Floer homology groups over $\operatorname{spin}^{c}$ structures in the fiber of $\mathfrak{s}_{k}$ under a certain surjective map $\operatorname{Spin}^{c}\left(Y_{0}(K)\right) \rightarrow \operatorname{Spin}^{c}\left(Y_{n}(K)\right)$ (see [5]). Our object is to use knot Floer homology to understand each term in this sequence, and therefore we quickly review the relevant facts about knot Floer homology (for details, see [8], 9] ).

Given $K \subset Y$, let $E$ denote the torus boundary of a regular neighborhood of $K$. One can then find a Heegaard surface for $Y$ of the form $E \# \Sigma_{g-1}$, with attaching circles $\boldsymbol{\alpha}=\alpha_{1}, \ldots, \alpha_{g}$ and $\boldsymbol{\beta}=\beta_{1}, \ldots, \beta_{g}$ where $\beta_{1} \subset E$ is a meridian for $K$ and $\left(\Sigma, \boldsymbol{\alpha}, \beta_{2}, \ldots, \beta_{g}\right)$ is a Heegaard diagram for the knot complement $Y \backslash K$. Let $w$ and $z$ denote a pair of basepoints, one on each side of the meridian $\beta_{1}$. The data $(E \# \Sigma, \boldsymbol{\alpha}, \boldsymbol{\beta}, w)$ together with a choice of $\operatorname{spin}^{c}$ structure $\mathfrak{s}$ on $Y$ can be used to define the Heegaard Floer chain groups $C F^{\infty}(Y, \mathfrak{s})$. The additional basepoint $z$, along with a choice of "relative $\operatorname{spin}^{c}$ structure" $\underline{\mathfrak{s}} \in \operatorname{Spin}^{c}\left(Y_{0}(K)\right)$ lifting $\mathfrak{s}$ gives rise to a filtration $\mathcal{F}$ on $C F^{\infty}(Y, \mathfrak{s})$. The "knot chain complex" $C F K^{\infty}(Y, K, \underline{\mathfrak{s}})$ is this filtered complex.

More concretely, we fix a Seifert surface $F$ for $K$ : then $F$ specifies the zeroframing on $K$, and can be capped off to a closed surface $\hat{F}$ in the zero-surgery $Y_{0}(K)$. The generators of $C F K^{\infty}(Y, K, F)$ are triples $[\mathbf{x}, i, j]$, where $\mathbf{x}$ denotes an intersection point between the $g$-dimensional tori $T_{\alpha}=\alpha_{1} \times \cdots \times \alpha_{g}$ and $T_{\beta}=$ $\beta_{1} \times \cdots \times \beta_{g}$ in the symmetric power $\operatorname{Sym}^{g}(E \# \Sigma)$, and $i$ and $j$ are integers. The point $\mathbf{x}$ along with the basepoint $w$ determine a $\operatorname{spin}^{c}$ structure $\mathfrak{s}_{w}(\mathbf{x})$ on $Y$ as well as a relative $\operatorname{spin}^{c}$ structure $\underline{\mathfrak{s}}_{w}(\mathbf{x})$; we require that $\mathfrak{s}_{w}(\mathbf{x})=\mathfrak{s}$. Furthermore, $i$ and $j$ are required to satisfy the equation

$$
\left\langle c_{1}\left(\underline{\mathfrak{s}}_{w}(\mathbf{x})\right),[\hat{F}]\right\rangle=2(j-i) .
$$

In this notation, the filtration $\mathcal{F}$ is simply $\mathcal{F}([\mathbf{x}, i, j])=j$; changing the Seifert surface $F$ shifts $\mathcal{F}$ by a constant. 
The boundary map $\partial^{\infty}$ in $C F K^{\infty}$ is defined by counting holomorphic disks in $\operatorname{Sym}^{g}(E \# \Sigma)$ and can only decrease the integers $i$ and $j$. Thus, for example, the subgroup $C\{i<0\}$ of $C F K^{\infty}$ generated by those $[\mathbf{x}, i, j]$ having $i<0$ is a subcomplex, and indeed is simply $C F^{-}(Y, \mathfrak{s})$ with an additional filtration. The quotient of $C F K^{\infty}$ by $C\{i<0\}$ is written $C\{i \geq 0\}$, and is a filtered version of $C F^{+}(Y, \mathfrak{s})$. We will use other similar notations to indicate other sub- or quotient complexes of $C F K^{\infty}$. In particular, $C F K^{0, *}$ is by definition the quotient complex $C\{i=0\}$, and $\widehat{H F K}$ is the homology of the graded object associated to the filtration $\mathcal{F}$ of $C F K^{0, *}$. We denote by $\widehat{H F K}(Y, K ; j)$ the summand of this group supported in filtration level $j$ (typically suppressing the $\operatorname{spin}^{c}$ structure from the notation).

As an additional piece of structure, we have a natural chain endomorphism $U$ on $C F K^{\infty}$ given by $U:[\mathbf{x}, i, j] \mapsto[\mathbf{x}, i-1, j-1]$.

Ozsváth and Szabó prove (Theorem 4.4 of $[\underline{8}$ ):

Theorem 3. For all sufficiently large positive $n$, there exists a $U$-equivariant isomorphism of chain complexes

$$
{ }^{b} \Psi^{+}: C F^{+}\left(Y_{n}, k\right) \rightarrow C\{i \geq 0 \text { or } j \geq k\}
$$

In particular, $H F^{+}\left(Y_{n}(K), k\right)$ is given by the homology of the portion of the knot complex indicated on the right-hand side. It is important to note that the proof of the above theorem shows that the stated identification is induced by a chain map coming from a particular $\operatorname{spin}^{c}$ structure on the cobordism $-W_{n}$ connecting $Y_{n}(K)$ to $Y$, namely the structure $\mathfrak{r}_{0} \in \operatorname{Spin}^{c}\left(-W_{n}\right)$ satisfying

$$
\left\langle c_{1}\left(\mathfrak{r}_{0}\right),[\hat{F}]\right\rangle=n-2 k \text {. }
$$

By contrast, the homomorphism $H^{+}\left(Y_{n}(K), k\right) \rightarrow H F^{+}(Y)$ in the long exact sequence (2) is given by the sum of the homomorphisms induced by all $\operatorname{spin}^{c}$ structures on $-W_{n}$ that restrict to the given $\operatorname{spin}^{c}$ structures on $Y_{n}(K)$ and $Y$. We will return to this point shortly.

Examining the sequence (2) again, we can now understand two of the groups appearing in terms of the knot chain complex: the theorem above identifies $\mathrm{HF}^{+}\left(Y_{n}, k\right)$, while $\mathrm{HF}^{+}(Y)$ is simply $H_{*}(C\{i \geq 0\})$, the homology of the complex obtained by forgetting the filtration. Note that under these identifications, the map $\mathrm{HF}^{+}\left(Y_{n}, k\right) \rightarrow$ $H F^{+}(Y)$ induced by $\mathfrak{r}_{0}$ as above corresponds to the map on homology induced by the natural projection $C\{i \geq 0$ or $j \geq k\} \rightarrow C\{i \geq 0\}$. Supposing the connecting homomorphism $\mathrm{HF}^{+}(Y) \rightarrow H F^{+}\left(Y_{0}(K),[k]\right)$ to be trivial, and ignoring the issue raised in the previous paragraph, we anticipate an isomorphism

$$
H F^{+}\left(Y_{0}(K),[k]\right) \cong H_{*}(C\{i<0 \text { and } j \geq k\}) \text {. }
$$

Indeed, it is implicit in [8] and described concretely in [2] that if $H F_{r e d}^{+}(Y)=0$ (and certain other, less important, hypotheses hold) then the connecting homomorphism is in fact trivial. We will show that the isomorphism holds even in certain cases when the connecting homomorphism is nontrivial.

Theorem 4. Fix a torsion spin ${ }^{c}$ structure $\mathfrak{s}$ on $Y$, and let $H F^{+}(Y, \mathfrak{s} ; A), H F^{+}\left(Y_{n}(K), k ; A\right)$, and $\mathrm{HF}^{+}\left(Y_{0}(K),[k] ; A\right)$ denote Floer homology groups in spin ${ }^{c}$ structures as above, with coefficients in a ring $A$. Assume that $k$ is nonzero, and make the following additional assumptions:

1. $\operatorname{HF}^{+}(Y, \mathfrak{s}, A)$ is a free A-module. 
2. The filtration on $H F_{r e d}(Y)$ is proportional to the degree: specifically, there is a constant $c$ such that the part of $H F_{r e d}(Y)$ that lies in filtration level $j$ is supported in absolute grading $j+c$.

3. For $F: H F^{+}\left(Y_{n}(K), k ; A\right) \rightarrow H F^{+}(Y ; A)$ the homomorphism in the long exact sequence (2), we have

$$
\operatorname{Ext}_{A}^{1}(\operatorname{ker}(F), \operatorname{coker}(F))=0 .
$$

4. The reduced homology $H F_{\text {red }}\left(Y_{n}(K), k, A\right)$ is supported in degrees at most $k+c-d$, where $d$ is the degree shift induced by the spin ${ }^{c}$ structure $\mathfrak{r}_{0}$ as in (3).

Then there is an identification of $A$-modules

$$
H F^{+}\left(Y_{0}(K),[k] ; A\right) \cong H_{*}(C\{i<0 \text { and } j \geq k\} ; A) .
$$

In particular hypotheses 1 and 3 of the theorem hold if $A$ is a field. If $A=\mathbb{Z}$, these hypotheses hold if, for example, $\mathrm{HF}^{+}(Y)$ and coker $(F)$ are torsion-free groups.

For the proof we need to analyze the surgery exact sequence:

$$
\cdots \stackrel{G}{\longrightarrow} H F^{+}\left(Y_{0}(K),[k]\right) \stackrel{H}{\longrightarrow} H F^{+}\left(Y_{n}, k\right) \stackrel{F}{\longrightarrow} H F^{+}(Y) \stackrel{G}{\longrightarrow} \cdots
$$

In particular, as mentioned above, we study the homomorphism $F$. Recall that since the $\operatorname{spin}^{c}$ structures $\mathfrak{s}$ and $\mathfrak{s}_{k}$ are torsion, the corresponding Heegaard Floer homology groups admit a (rational-valued) grading that lifts the natural relative $\mathbb{Z}$ grading. A $\operatorname{spin}^{c}$ structure $\mathfrak{r}$ on $-W_{n}$ extending $\mathfrak{s}$ restricts to $\mathfrak{s}_{k}$ on $Y_{n}(K)$ if and only if it satisfies

$$
\left\langle c_{1}(\mathfrak{r}),[\hat{F}]\right\rangle=n-2 k+2 x n
$$

for some integer $x \in \mathbb{Z}$, where $\hat{F}$ denotes the capped-off Seifert surface in $-W_{n}$ as before. Recall that we assume $k \in\{1, \ldots, n-1\}$. Now, $F$ is the sum of the maps $F_{-W_{n}, \mathfrak{r}}$ for $\mathfrak{r}$ corresponding to all values of $x$. According to [6], the map $F_{\mathfrak{r}}: H F^{+}\left(Y_{n}(K), k\right) \rightarrow H F^{+}(Y)$ induced by $\mathfrak{r}$ shifts degree by the quantity

$$
\operatorname{deg}\left(F_{\mathfrak{r}}\right)=-n x^{2}-(n-2 k) x+\frac{n-(n-2 k)^{2}}{4 n} .
$$

The maximum value of this degree occurs when $x$ is the closest integer to $-\frac{1}{2}+\frac{k}{n}$, which, given our assumption on $k$, is $x=0$. Comparing with (3) we see that the $\operatorname{spin}^{c}$ structure $\mathfrak{r}_{0}$ corresponding to $x=0$ is both the "leading order term" (i.e., the homogeneous part with maximal degree) in the homomorphism $F$ and also the $\operatorname{spin}^{c}$ structure inducing the identification of $\mathrm{HF}^{+}\left(Y_{n}(K), k\right)$ with $H_{*}(C\{i \geq$ 0 or $j \geq k\}$ ).

With the above in mind, we write (as in 8]) $F=f_{1}+f_{2}$ where $f_{1}$ is the homogeneous part with highest degree (that is, $f_{1}=F_{-W_{n}, \mathfrak{r}_{0}}$ ) and $f_{2}$ is the sum of all lower-degree parts of $F$. Note that the highest-degree part of $f_{2}$ has degree equal to $\operatorname{deg}\left(f_{1}\right)-\min \{2 k, 2(n-k)\}$.

Lemma 5. Suppose there exists a homomorphism $R: \operatorname{Im}\left(f_{2}\right) \rightarrow H F^{+}\left(Y_{n}(K), k\right)$ satisfying $f_{1} \circ R=i d$ on $\operatorname{Im}\left(f_{2}\right)$ (that is, $R$ is a partially-defined right inverse for $\left.f_{1}\right)$. Then there is an isomorphism $g: H F^{+}\left(Y_{n}(K), k\right) \rightarrow H F^{+}\left(Y_{n}(K), k\right)$ such that

$$
f_{1}=F \circ g .
$$

In particular, $\operatorname{coker}(F)=\operatorname{coker}\left(f_{1}\right)$, and $\operatorname{ker}(F) \cong \operatorname{ker}\left(f_{1}\right)$ (via the map $g$ ). 
Proof. Since $F=f_{1}+f_{2}$, we have

$$
F=f_{1}\left(1+R f_{2}\right) .
$$

From the remarks on the degree shift above, the composition $R f_{2}$ is strictly decreasing in degree. Since $H F^{+}\left(Y_{n}(K), k\right)$ is trivial in sufficiently low degrees, the sum

$$
g=\left(1+R f_{2}\right)^{-1}=\sum_{n \geq 0}\left(-R f_{2}\right)^{n}
$$

is finite, and hence $g$ is the desired isomorphism.

If we can construct the right inverse $R$ as in the lemma, Theorem 4 will follow easily. Indeed, let us identify $H F^{+}\left(Y_{n}(K), k\right)$ with $H_{*}(C\{i \geq 0$ or $j \geq k\})$ as in Theorem 3 then we've seen that $f_{1}$ is identified with the natural projection

$$
\pi_{*}: H_{*}(C\{i \geq 0 \text { or } j \geq k\}) \rightarrow H_{*}(C\{i \geq 0\})=H F^{+}(Y) .
$$

Hence we have a commutative diagram

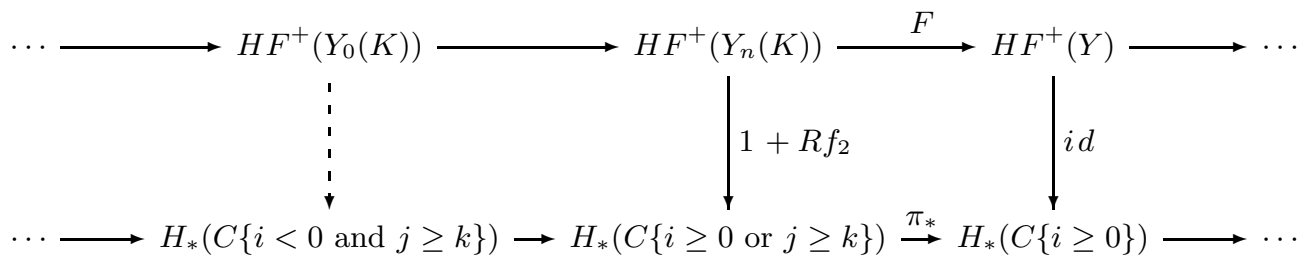

where the solid vertical arrows are isomorphisms and the top row is the surgery long exact sequence (5). We have not constructed a map corresponding to the dashed arrow, but the above diagram allows us to write another:

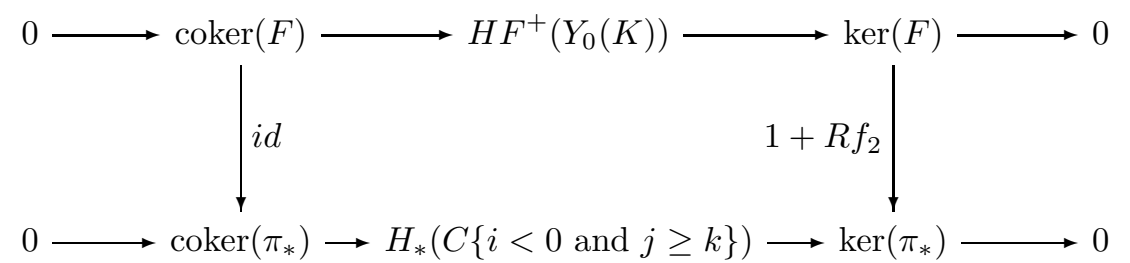

Again the vertical arrows are isomorphisms, so that $H^{+}\left(Y_{0}(K)\right)$ and $H_{*}(C\{i<$ 0 and $j \geq k\}$ ) are both extensions of $\operatorname{coker}(F)$ by $\operatorname{ker}(F)$. Under hypothesis 3 of the theorem such an extension is unique, which completes the proof of Theorem 4 .

It remains to construct the right inverse $R$.

Lemma 6. Under hypotheses (1), (2), and (4) of Theorem 4 there exists a right inverse $R$ for $f_{1}$ that is defined on $\operatorname{Im}\left(f_{2}\right)$.

Note that the only place that hypothesis 3 of the theorem is required is at the last step of its proof (see above).

Proof. Since $\operatorname{Im}\left(f_{1}\right) \subset H F^{+}(Y)$ is free by hypothesis, we can find a right inverse $R$ : $\operatorname{Im}\left(f_{1}\right) \rightarrow H F^{+}\left(Y_{n}(K), k\right)$. We need to check that $R$ is defined on $\operatorname{Im}\left(f_{2}\right)$, i.e., that $\operatorname{Im}\left(f_{2}\right) \subset \operatorname{Im}\left(f_{1}\right)$. We identify $H F^{+}(Y)$ with $H_{*}(C\{i \geq 0\})$ and $H F^{+}\left(Y_{n}(K), k\right)$ with $H_{*}(C\{i \geq 0$ or $j \geq k\})$, so that $f_{1}$ corresponds to the projection $\pi_{*}$ as above.

Recall that because of the structure of the chain complex $C F^{\infty}(Y)$ the image of the action of $U^{r}$ on $H^{+}(Y)$ is independent of $r$ for sufficiently large $r$. By 
definition the reduced Floer homology group $\mathrm{HF}_{\text {red }}(Y)$ is the quotient of $\mathrm{HF}^{+}(Y)$ by $\operatorname{Im}\left(U^{r}\right)$ for any such large $r$ : in other words there is an exact sequence

$$
0 \longrightarrow \operatorname{Im}\left(U^{r}\right) \longrightarrow H F^{+}(Y) \longrightarrow H F_{r e d}(Y) \longrightarrow 0 \text {. }
$$

The knot filtration induces a filtration on $H F_{\text {red }}$.

Now, since the chain complexes $C\{i \geq 0\}$ and $C\{i \geq 0$ or $j \geq k\}$ are identical for sufficiently large degrees, it is clear that $f_{1}=\pi_{*}$ maps onto $\operatorname{Im}\left(U^{r}\right)$ for $r>>0$. We claim that $f_{1}$ also maps onto $H_{\text {red }}^{<k}(C\{i \geq 0\})$, where $H_{\text {red }}^{<k}$ denotes that portion of the reduced homology that lies in filtration level $j=k-1$ or below. To see this, it suffices to show that the connecting homomorphism $\delta: H_{*}(C\{i \geq 0\}) \rightarrow$ $H_{*}(C\{i<0$ and $j \geq k\})$ is trivial on the indicated group. But for $x \in C\{i \geq 0\}$ a cycle, $\delta x$ is given by the portion of $\partial^{\infty} x$ that lies in $C\{i<0$ and $j \geq k\}$, where $\partial^{\infty}$ is the boundary map in $C F K^{\infty}$. The statement follows since $\partial^{\infty}$ is nonincreasing in $j$.

From the discussion above, $f_{1}$ maps onto all summands of $\mathrm{HF}^{+}(Y)$ that lie in degree less than $k+c$ as well as $\operatorname{Im}\left(U^{r}\right)$ (in all degrees). By $U$-equivariance $f_{2}$ maps $\operatorname{Im}\left(U^{r}\right)$ into $\operatorname{Im}\left(U^{r}\right)$, so we need only check that $f_{2}$ maps $H F_{r e d}\left(Y_{n}(K), k\right)$ into $\operatorname{Im}\left(f_{1}\right)$. But this follows immediately from the facts that $f_{2}$ has degree strictly less than that of $f_{1}$ and that $H F_{r e d}\left(Y_{n}\right)$ is supported in degrees $\leq k+c-\operatorname{deg}\left(f_{1}\right)$.

\section{Calculation for Separating Twists}

3.1. Preliminaries: Calculation of Knot Homology. A surgery diagram for the mapping torus $M\left(t_{\sigma}^{n}\right)$ is obtained from one for $\Sigma_{g} \times S^{1}$ by adding $n$ parallel copies of the separating curve $\sigma$ with surgery coefficient -1 (here and subsequently we assume $n>0$; the case of negative $n$ is entirely parallel and will be described later). In particular, $M\left(t_{\sigma}^{n}\right)$ can be obtained from 0-surgery along a knot $\tilde{K}$ in a connected sum $\#^{2 g-2}\left(S^{1} \times S^{2}\right) \# M_{n}$, where $M_{n}$ is described below. The situation is illustrated for $g=3$ and $n=1$ in Figure 1

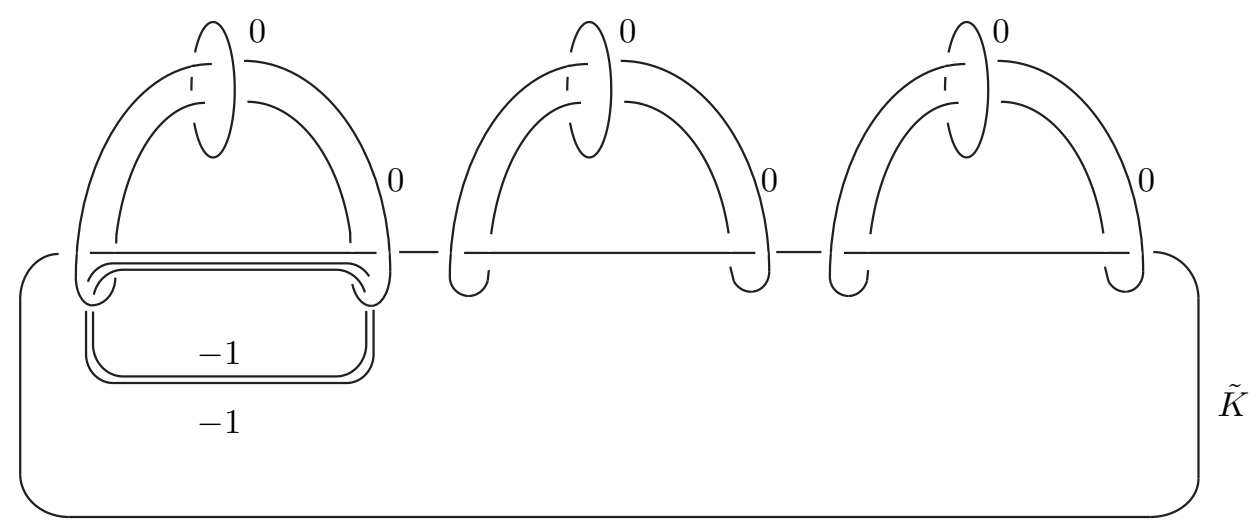

Figure 1 . The knot $\tilde{K}$ in the case $g=3$ and $n=2$.

We can think of $\tilde{K}$ as a connected sum of knots: write $B(p, q)$ for the knot given as the third component of the Borromean rings after performing surgery on the other two components with surgery coefficients $p$ and $q$. Then $\widetilde{K}=\#^{g-1} B(0,0) \# K$, where $K$ is the knot indicated in Figure 2 


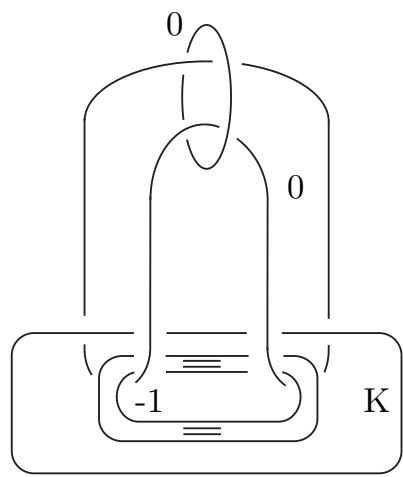

(a)

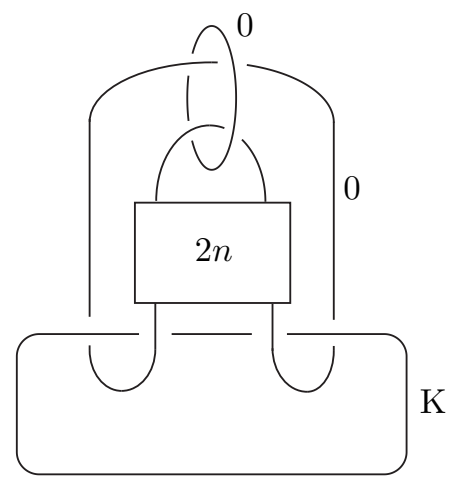

(b)

Figure 2. Two equivalent pictures for the $\operatorname{knot} K$ in $M_{n}$. In picture (a) there are $n$ parallel -1 circles. The $2 n$ in picture (b) indicates $2 n$ positive half-twists.

We denote by $M_{n}$ the manifold containing $K$, so $M_{n}$ is obtained by performing 0framed surgery on two components of the Borromean rings and -1 framed surgery on each of $n$ parallel copies of the third component. By blowing down all the -1 circles, we can see $M_{n}$ as the result of 0 -surgery on both components of an " $n$-clasped Whitehead link" as shown in figure 2 (b) (the $2 n$ in the box denotes $2 n$ positive half-twists). As a preliminary to the calculation of the knot homology of $K$, we find the homology $\widehat{H F}\left(M_{n}\right)$.

Let $Z$ denote the 3 -manifold that results from changing the surgery coefficient from 0 to -1 on the smaller 0 -framed circle in the diagram for $M_{n}$. An easy isotopy shows that after blowing down this -1 circle, $Z$ is given as 0 -framed surgery on the pretzel knot $P(-2 n+1,-1,-1)$ (here we follow the notation conventions of [7], whereby $P(1,1,1)$ is the right-handed trefoil). It follows from the calculations in section 8 of [7] that for the torsion $\operatorname{spin}^{c}$ structure $\mathfrak{s}_{0}$,

$$
H F_{k}^{+}\left(Z ; \mathfrak{s}_{0}\right)= \begin{cases}\mathbb{Z} & k \equiv 1 / 2 \bmod \mathbb{Z} \text { and } k \geq 3 / 2 \\ \mathbb{Z}^{n} & k=1 / 2 \\ 0 & \text { else }\end{cases}
$$

and that the image of $U$ in degree $1 / 2$ is nontrivial.

Remark 7. For the rest of this section and throughout the next, we take coefficients in the ring $A=\mathbb{Z}$. In particular it suffices for the verification of hypothesis (3) of Theorem 4 to show that $\operatorname{coker}(F)$ is a free abelian group.

With this information, the long exact sequence in Floer homology connecting $S^{1} \times S^{2}, Z$, and $M_{n}$ immediately gives

$$
H F_{k}^{+}\left(M_{n} ; \mathfrak{s}_{0}\right)= \begin{cases}\mathbb{Z}^{2} & k \equiv 1 \bmod \mathbb{Z} \text { and } k \geq 1 \\ \mathbb{Z}^{n+1} & k=0 \\ 0 & \text { else }\end{cases}
$$

From this we infer $\widehat{H F}\left(M_{n}\right)=\mathbb{Z}_{(1)}^{n+1} \oplus \mathbb{Z}_{(0)}^{n+1}$. 
We are interested in the knot Floer homology $\widehat{H F K}\left(M_{n}, K\right)$. The following was proved in 2]:

Lemma 8. The knot Floer homology groups for $\left(M_{1}, K\right)$ are given by

$$
\widehat{\operatorname{HFK}}\left(M_{1}, K ; j\right)= \begin{cases}\mathbb{Z}_{(1)} & j=1 \\ \mathbb{Z}_{(0)}^{3} \oplus \mathbb{Z}_{(1)} & j=0 \\ \mathbb{Z}_{(-1)} & j=-1 \\ 0 & \text { otherwise }\end{cases}
$$

The spectral sequence that calculates $\widehat{H F}\left(M_{1}\right)$ from $\widehat{H F K}\left(M_{1}, K\right)$ collapses at the $E_{2}$ level, and the only nontrivial differential is a surjection $d_{1}: \mathbb{Z}_{(0)}^{3} \rightarrow \mathbb{Z}_{(-1)}$.

We now calculate the result for the case of general $n$ :

Proposition 9. The knot Floer homology groups of $K$ in $M_{n}$ are given by

$$
\widehat{H F K}\left(M_{n}, K ; j\right)= \begin{cases}\mathbb{Z}_{(1)} & j=1 \\ \mathbb{Z}_{(0)}^{n+2} \oplus \mathbb{Z}_{(1)}^{n} & j=0 \\ \mathbb{Z}_{(-1)} & j=-1 \\ 0 & \text { otherwise }\end{cases}
$$

The only nontrivial differential in the spectral sequence converging to $\widehat{H F}\left(M_{n}\right)$ is a surjection $d_{1}: \mathbb{Z}_{(0)}^{n+2} \rightarrow \mathbb{Z}_{(-1)}$.

Proof. We proceed inductively: suppose $n \geq 2$. We look at a surgery sequence arising from figure 2(a). Choose one of the -1 circles in the picture for $M_{n}$ and let $A$ denote the 3 -manifold obtained by changing the -1 to 0 . Then the surgery sequence appears as

$$
\cdots \rightarrow \widehat{H F K}\left(M_{n-1}, K ; j\right) \rightarrow \widehat{H F K}\left(M_{n}, K ; j\right) \rightarrow \widehat{H F K}(A, K ; j) \rightarrow \cdots
$$

Now $(A, K)$ is unknotted since $K$ can slide over the 0 -framed circle. Therefore the filtration induced by $K$ on $\widehat{C F}(A)$ is trivial, and hence $\widehat{H F K}(A, K)$ is supported in level $j=0$. It follows immediately that the group $\widehat{H F K}\left(M_{n}, K ; j\right)$ has the claimed form when $j \neq 0$.

For the case $j=0$, note that the calculation so far together with the structure of $\widehat{H F}\left(M_{n}\right)$ already imply that $\widehat{H F K}\left(M_{n}, K ; 0\right)$ is supported in degrees 0 and 1 , by consideration of the spectral sequence for $\widehat{H F}$. It then follows that the only nontrivial differential in the latter spectral sequence is $d_{1}$, and in fact $d_{1}: \widehat{H F K}\left(M_{n}, K ; 0\right) \rightarrow \widehat{H F K}\left(M_{n}, K,-1\right) \cong \mathbb{Z}$ must be surjective. An argument similar to the case $n=1$ (see 2]) together with our inductive knowledge of $\widehat{H F K}\left(M_{n-1}, K\right)$ shows that $d_{1}: \widehat{H F K}\left(M_{n}, K, 1\right) \rightarrow \widehat{H F K}\left(M_{n}, K ; 0\right)$ is trivial. The proposition follows from this and the fact that $\widehat{H F}\left(M_{n}\right)=\mathbb{Z}_{(1)}^{n+1} \oplus \mathbb{Z}_{(0)}^{n+1}$.

It will be convenient in what follows to write $\widehat{H F K}\left(M_{n}, K\right)$ as

$$
\widehat{H F K}\left(M_{n}, K\right) \cong \Lambda^{*} H^{1}\left(\Sigma_{1}\right) \oplus H^{*}\left(\coprod_{n} S^{1}\right) .
$$

In the above, the grading on $\Lambda^{*} H^{1}\left(\Sigma_{1}\right)$ is "centered," meaning that $\Lambda^{i} H^{1}\left(\Sigma_{g}\right)$ is considered to have grading $i-g$. The grading on the second factor above is the usual homological grading. The filtration is not evident from the notation, however 
we see that it is equal to the (centered) grading on the first factor while the second factor lies in filtration level 0 .

In this notation, we can express the single nontrivial differential in the spectral sequence for $\widehat{H F}\left(M_{n}\right)$ as the map $\Lambda^{1} H^{1}\left(\Sigma_{1}\right) \rightarrow \Lambda^{0} H^{1}\left(\Sigma_{1}\right)$ given by contraction with a generator $\gamma$ of $H_{1}\left(\Sigma_{1}\right)$, which we represent as an embedded circle in the torus also denoted $\gamma$.

The connected sum theorem for $\widehat{H F K}$ then gives:

Proposition 10. The knot Floer homology of $\tilde{K} \subset Y=M_{n} \#^{2 g-2}\left(S^{1} \times S^{2}\right)$ is given by

$$
\widehat{H F K}(Y, \tilde{K})=\Lambda^{*} H^{1}\left(\Sigma_{g}\right) \oplus\left[\Lambda^{*} H^{1}\left(\Sigma_{g-1}\right) \otimes H^{*}\left(\coprod_{n} S^{1}\right)\right]
$$

The only nontrivial differential in the spectral sequence for $\widehat{H F}(Y)$ is given by contraction with a generator $\gamma \in H_{1}\left(\Sigma_{g}\right)$ in the first summand above.

Indeed, it is shown in 8 that $\widehat{H F K}(B(0,0)) \cong \Lambda^{*} H^{1}\left(\Sigma_{1}\right)$ with centered grading. Formula (7) follows from this and the Künneth formula for $\widehat{H F K}$ under connected sum [8].

3.2. Verification of Hypotheses. Knowledge of the knot Floer homology $\widehat{H F K}(Y, K)$ can often lead to understanding of the full Heegaard Floer groups $\mathrm{HF}^{+}$for $Y$ and the surgered manifold $Y_{n}(K)$. Indeed, there is a spectral sequence for $H F^{\infty}(Y)$ associated to the filtration of $C F K^{\infty}(Y, K)$ given by $[\mathbf{x}, i, j] \mapsto i+j$, whose $E_{1}$ term is $\widehat{H F K}(Y, K) \otimes \mathbb{Z}\left[U, U^{-1}\right]$. The $d_{1}$ differential is a sum of homomorphisms that map the group at position $(i, j)$ in $C F K^{\infty}$ to those at positions $(i-1, j)$ and $(i, j-1)$ : the "vertical" and "horizontal" components of $d_{1}$. These components can in turn be determined from the spectral sequence for $\widehat{H F}(Y)$ coming from $C F K^{0, *}$ : indeed, the vertical component is precisely (after a translation by a power of $U$ ) the first differential in the latter sequence. On the other hand the complex $C F K^{*, 0}$ can also be identified with a filtered version of $\widehat{C F}$, so the horizontal component of $d_{1}$ is also determined by the differential in the spectral sequence calculating $\widehat{H F}$ from $\widehat{H F K}$. We put these ideas to work in understanding the Floer homologies of the particular $Y$ and $Y_{n}$ relevant to our situation.

We begin by determining the differentials in the spectral sequence for $C F^{\infty}$, in the case of $(Y, \tilde{K})$ as in Proposition [10] As noted above, the $E_{1}$ term is given by $\widehat{H F K}(Y, \tilde{K}) \otimes \mathbb{Z}\left[U, U^{-1}\right]$. Explicitly, this is

$$
E_{1}=\left(\Lambda^{*} H^{1}\left(\Sigma_{g}\right) \otimes \mathbb{Z}\left[U, U^{-1}\right]\right) \oplus\left(\Lambda^{*} H^{1}\left(\Sigma_{g-1}\right) \otimes H^{*}\left(\coprod_{n} S^{1}\right) \otimes \mathbb{Z}\left[U, U^{-1}\right]\right) .
$$

The $d_{1}$ differential is nontrivial only on the first summand, where its action is described as follows. Decompose $\Sigma_{g}=\Sigma_{1} \# \Sigma_{g-1}$ where the generator $\gamma \in H_{1}\left(\Sigma_{1}\right)$ of Proposition 10 is contained in the first factor. Let

$$
\begin{aligned}
& E_{+}=\left(\Lambda^{0} H^{1}\left(\Sigma_{1}\right) \oplus \Lambda^{2} H^{1}\left(\Sigma_{1}\right)\right) \otimes \Lambda^{*} H^{1}\left(\Sigma_{g-1}\right) \otimes \mathbb{Z}\left[U, U^{-1}\right] \\
& E_{-}=\Lambda^{1} H^{1}\left(\Sigma_{1}\right) \otimes \Lambda^{*} H^{1}\left(\Sigma_{g-1}\right) \otimes \mathbb{Z}\left[U, U^{-1}\right],
\end{aligned}
$$

so that $E_{+} \oplus E_{-}$is isomorphic to the first summand of the $E_{1}$ term above. Then one can check just as in [8] or 2] that the $d_{1}$ differential is trivial on $E_{+}$while on 
$E_{-}$it is given by

$$
d_{1}\left(\omega \otimes U^{j}\right)=\iota_{\gamma} \omega \otimes U^{j}+P D(\gamma) \wedge \omega \otimes U^{j+1} .
$$

It is a straightforward exercise to check that the homology of this differential is given by

$$
\begin{aligned}
H\left(E_{+} \oplus E_{-}, d_{1}\right)= & \Lambda^{0} H^{1}\left(\Sigma_{1}\right) \otimes \Lambda^{*}\left(\Sigma_{g-1}\right) \otimes \mathbb{Z}\left[U, U^{-1}\right] \\
& \oplus P D(\gamma) \wedge \Lambda^{*} H^{1}\left(\Sigma_{g-1}\right) \otimes \mathbb{Z}\left[U, U^{-1}\right] \\
\cong & \Lambda^{*} H^{1}\left(\Sigma_{g-1}\right) \otimes H^{*}\left(S^{1}\right) \otimes \mathbb{Z}\left[U, U^{-1}\right] .
\end{aligned}
$$

Therefore the second term in our spectral sequence appears as

$$
\begin{aligned}
E_{2}= & \left(\Lambda^{*} H^{1}\left(\Sigma_{g-1}\right) \otimes H^{*}\left(S^{1}\right) \otimes \mathbb{Z}\left[U, U^{-1}\right]\right) \\
& \oplus\left(\Lambda^{*} H^{1}\left(\Sigma_{g-1}\right) \otimes H^{*}\left(\coprod_{n} S^{1}\right) \otimes \mathbb{Z}\left[U, U^{-1}\right]\right) \\
= & \Lambda^{*} H^{1}\left(\Sigma_{g-1}\right) \otimes H^{*}\left(\coprod_{n+1} S^{1}\right) \otimes \mathbb{Z}\left[U, U^{-1}\right] .
\end{aligned}
$$

Observe that there is an isomorphism of $\mathbb{Z}[U]$-modules $H^{*}\left(S^{1} \amalg S^{1}\right) \otimes \mathbb{Z}\left[U, U^{-1}\right] \cong$ $\Lambda^{*} H^{1}\left(\Sigma_{1}\right) \otimes \mathbb{Z}\left[U, U^{-1}\right]$. Therefore we can write the above as

$$
E_{2}=\left(\Lambda^{*} H^{1}\left(\Sigma_{g}\right) \otimes \mathbb{Z}\left[U, U^{-1}\right]\right) \oplus\left(\Lambda^{*} H^{1}\left(\Sigma_{g-1}\right) \otimes H^{*}\left(\coprod_{n-1} S^{1}\right) \otimes \mathbb{Z}\left[U, U^{-1}\right]\right),
$$

where we have written $\Lambda^{*} H^{1}\left(\Sigma_{g-1}\right) \otimes \Lambda^{*} H^{1}\left(\Sigma_{1}\right)=\Lambda^{*} H^{1}\left(\Sigma_{g}\right)$.

We must now determine subsequent differentials in the spectral sequence, if any. Note first that if $n=1$ then the above reduces to $\Lambda^{*} H^{1}\left(\Sigma_{g}\right) \otimes \mathbb{Z}\left[U, U^{-1}\right] \cong$ $H F^{\infty}(Y)$. Indeed, this identification follows using the connected sum theorem for $H F^{\infty}$ (recall that $\left.Y=M_{n} \#(2 g-2) S^{1} \times S^{2}\right)$ and the fact that since $b_{1}\left(M_{n}\right)=2$, the Floer homology $H F^{\infty}\left(M_{n}\right)$ is "standard" (see [5], 7]). Thus when $n=1$ there are no subsequent differentials, and in fact this case has already been understood in 2 .

In general, the $d_{2}$ differential is a sum of three terms, mapping $C\{i, j\}$ into $C\{i-2, j\} \oplus C\{i-1, j-1\} \oplus C\{i, j-2\}$. In our case, however, the vertical and horizontal components must be trivial because those (just as in the case of $d_{1}$ ) correspond to differentials in the second term of the spectral sequence for $\widehat{H F}$, which we have seen collapses at the second term. Therefore $d_{2}$ must be given by a $\operatorname{map}_{2}: E_{2}\{i, j\} \rightarrow E_{2}\{i-1, j-1\}$.

Lemma 11. In terms of the expression (10), $d_{2}$ is trivial on the first factor and acts on the second factor by an isomorphism

$$
\Lambda^{*} H^{1}\left(\Sigma_{g-1}\right) \otimes H^{0}\left(\coprod_{n-1} S^{1}\right) \otimes U^{k} \longrightarrow \Lambda^{*}\left(\Sigma_{g-1}\right) \otimes H^{1}\left(\coprod_{n-1} S^{1}\right) \otimes U^{k+1} .
$$

All subsequent differentials in the spectral sequence are trivial.

Proof. We can see that further differentials must be trivial by examining the gradings. In (10), the exterior algebras are equipped with the centered grading, the grading on $H^{*}\left(S^{1}\right)$ is the natural homological grading, and $U$ is considered to have grading -2 . The filtration (the " $j$-coordinate") is equal to the grading on the exterior algebras and on $\mathbb{Z}\left[U, U^{-1}\right]$, but $H^{*}\left(\coprod_{n-1} S^{1}\right)$ is considered to lie in filtration 
level 0. (All of these observations can be deduced from the remarks after Propositions 9 and [0]) Finally, the " $i$-coordinate" is recovered by recalling that in the expression $E_{1}=\widehat{H F K} \otimes \mathbb{Z}\left[U, U^{-1}\right]$, the subgroup $\widehat{H F K} \otimes 1$ lies in the column $i=0$. It is now straightforward to see that if $(i+j)-\left(i^{\prime}+j^{\prime}\right)>2$ then there are no elements $a \in C\{i, j\}$ and $b \in C\left\{i^{\prime}, j^{\prime}\right\}$ whose degrees differ by 1 , so differentials beyond $d_{2}$ vanish for dimensional reasons.

It follows that the homology of $d_{2}$ must yield $H F^{\infty}(Y) \cong \Lambda^{*} H^{1}\left(\Sigma_{g}\right) \otimes \mathbb{Z}\left[U, U^{-1}\right]$; furthermore the only factors in $E_{2}\{i, j\}$ and $E_{2}\{i-1, j-1\}$ that can be connected by this differential (i.e., factors whose degrees differ by 1 ) are those that are indicated in the statment. Dimensional considerations ensure that the differential must be an isomorphism between those factors.

With this understanding of the differentials in the spectral sequence it is a straightforward matter to determine $\mathrm{HF}^{+}(Y)$ and $\mathrm{HF}^{+}\left(Y_{n}\right)$ to a degree sufficient to verify the hypotheses of Theorem 4 Indeed, hypotheses 1 and 2 of that theorem follow from:

Lemma 12. With $Y=M_{n} \#(2 g-2) S^{1} \times S^{2}$ as above, we have an identification of $\mathbb{Z}[U]$-modules

$$
H F^{+}(Y, \mathfrak{s})=\left(\Lambda^{*}\left(\Sigma_{g-1}\right) \otimes \mathbb{Z}\left[U^{-1}\right]\right) \oplus(n-1) \Lambda^{*} H^{1}\left(\Sigma_{g-1}\right),
$$

where $\mathfrak{s}$ denotes the torsion spin ${ }^{c}$ structure on $Y$, and $(n-1) \Lambda^{*} H^{1}\left(\Sigma_{g-1}\right)$ denotes the direct sum of $n-1$ copies of the exterior algebra. In particular, $H F_{\text {red }}(Y, \mathfrak{s})=$ $(n-1) \Lambda^{*} H^{1}\left(\Sigma_{g-1}\right)$ as graded groups (where the grading on the exterior algebra is centered as before), and the filtration on $H F_{r e d}(Y, \mathfrak{s})$ is equal to the grading.

Note that one can prove this (except for the information about the filtration) without using the spectral sequence by appealing to the connected sum theorem for Heegaard Floer homology.

Proof. By restriction, the filtration $\mathcal{F}:[\mathbf{x}, i, j] \mapsto i+j$ used to produce the spectral sequence for $H F^{\infty}$ also gives a filtration on $C F^{+}$and thereby a spectral sequence for $\mathrm{HF}^{+}$whose differentials are just the restrictions of the originals to this quotient complex. In particular the $E_{1}$ term appears as $\widehat{H F K}(Y, \tilde{K}) \otimes \mathbb{Z}\left[U^{-1}\right]$, with differential given by (9). One checks that no new cycles are created in $E_{1}$ by passing to the quotient complex $C\{i \geq 0\}$, so that the $E_{2}$ term here looks just like (10) with $\mathbb{Z}\left[U, U^{-1}\right]$ replaced by $\mathbb{Z}\left[U^{-1}\right]$.

The second differential takes the same form as previously, but in this case there are additional cycles in $E_{2}$ : since $d_{2}$ maps $E_{2}\{i, j\}$ into $E_{2}\{i-1, j-1\}$, it sends those elements lying in the group $\Lambda^{*} H^{1}\left(\Sigma_{g-1}\right) \otimes H^{0}\left(\coprod_{n-1} S^{1}\right) \otimes U^{0}$ (supported in the column $i=0$ ) to 0 , where it did not do so in the spectral sequence for $H F^{\infty}$. Hence this group is precisely $H F_{r e d}(Y, \mathfrak{s})$, and can be written $(n-1) \Lambda^{*} H^{1}\left(\Sigma_{g-1}\right)$ as a graded group. The lemma follows immediately, keeping in mind the structure of the filtration as described in the proof of Lemma 11

For the remaining hypotheses in Theorem 4 we must understand $H F^{+}\left(Y_{n}(\tilde{K}), k\right)$. Recall the isomorphism ${ }^{b} \Psi^{+}: C F^{+}\left(Y_{n}\right) \cong C\{i \geq 0$ or $j \geq k\}$ of Theorem 3 Using ${ }^{b} \Psi^{+}$, the filtration $\mathcal{F}$ on $C F^{\infty}(Y)$ restricts to a filtration on $C F^{+}\left(Y_{n}\right)$, and thereby gives a spectral sequence for $H F^{+}\left(Y_{n}\right)$ It is a simple matter to see, by examining the domains and ranges of the differentials, that $H F_{\text {red }}\left(Y_{n}\right)$ must be supported along the right-angled strip $\max \{i, j-k\}=0$. (Indeed, just as in the case of $Y$ 
the reduced homology is a result of the "additional" cycles for $d_{2}$ that arise from passing to the quotient complex $C\{i \geq 0$ or $j \geq 0\}$, which can only lie in the indicated region of the $(i, j)$ plane.) Since the $j$-coordinate measures the filtration, hypothesis 4 follows immediately.

Finally for hypothesis 3 , note that the proof of Theorem 4 shows that we may replace $F$ by $\pi_{*}$ since the corresponding kernels and cokernels are isomorphic once the other hypotheses hold (c.f. Lemmas 5 and 6). But it is clear that $\operatorname{ker} \pi_{*}$ is equal to that portion of $H_{*}(C\{i \geq 0$ or $j \geq k\})$ lying in the region $i<0$, while its image is $\operatorname{Im}\left(U^{r}\right) \oplus H F_{r e d}^{\leq k}(Y) \subset H F^{+}(Y)$. In particular

$$
\operatorname{coker}\left(\pi_{*}\right)=H F_{r e d}^{>k}(Y) \cong \Lambda^{>g-1+k} H^{1}\left(\Sigma_{g-1}\right)
$$

is a free $\mathbb{Z}$-module.

3.3. Calculation. We turn our attention to determining the Heegaard Floer homology groups $H F^{+}\left(M\left(t_{\sigma}^{n}\right), \mathfrak{s}\right)$ where $\mathfrak{s}$ is any nontorsion $\operatorname{spin}^{c}$ structure, $\sigma \subset \Sigma_{g}$ is a separating curve such that $\Sigma_{g} \backslash \sigma$ consists of components of genus 1 and $g-1$, $t_{\sigma}$ denotes the right-handed Dehn twist about $\sigma$, and $M\left(t_{\sigma}^{n}\right)$ is the mapping torus of the diffeomorphism $t_{\sigma}^{n}$ for any $n \neq 0$. We focus first on the case of $n>0$.

The result of the preceding section is that the desired Floer homology can be determined from the knot complex for $\tilde{K} \subset Y$ as $H_{*}(C\{i<0$ and $j \geq k\})$, where $\tilde{K}=K \#(g-1) B(0,0)$ as before. We assume here that $k>0$. We make use of the same spectral sequence as in that section to calculate this homology; note that the differentials have already been determined. Now, the $E_{1}$ term of this spectral sequence is just the portion of $\widehat{H F K}(Y, \tilde{K}) \otimes \mathbb{Z}\left[U, U^{-1}\right]$ that lies in the relevant part of the $(i, j)$ plane. Following [8] and [2], we introduce the notation

$$
X(g, d)=\bigoplus_{i=0}^{d} \Lambda^{2 g-i} H^{1}\left(\Sigma_{g}\right) \otimes_{\mathbb{Z}} \mathbb{Z}[U] / U^{d+1-i} .
$$

Thus $X(g, d) \cong H^{*}\left(\operatorname{Sym}^{d} \Sigma_{g}\right)$ as $\mathbb{Z}[U]$-modules (see [3] $)$. Then it is easy to see that in the spectral sequence for $H F^{+}\left(Y_{0}(K), \mathfrak{s}_{k}\right)$,

$$
E_{1}=X(g, d) \oplus\left[X(g-1, d-1) \otimes H^{*}\left(\coprod_{n} S^{1}\right)\right],
$$

where $d=g-1-k$. The $d_{1}$ differential is nontrivial only on the first factor (see the discussion after equation (8) ), where it acts as in equation (91). The complex $\left(X(g, d), d_{1}\right)$ was considered in 2], where it was shown that its homology is

$$
H_{*}\left(X(g, d), d_{1}\right)=\left(X(g-1, d-1) \otimes H^{*}\left(S^{1}\right)\right) \oplus \Lambda^{2 g-2-d} H^{1}\left(\Sigma_{g-1}\right)_{(g-d)} .
$$

Hence,

$$
\begin{aligned}
E_{2}= & {\left[X(g-1, d-1) \otimes H^{*}\left(S^{1}\right) \oplus \Lambda^{2 g-2-d} H^{1}\left(\Sigma_{g-1}\right)_{(g-d)}\right] } \\
& \oplus\left[X(g-1, d-1) \otimes H^{*}\left(\coprod_{n} S^{1}\right)\right] \\
= & {\left[X(g-1, d-1) \otimes H^{*}\left(S^{1} \sqcup S^{1}\right) \oplus \Lambda^{2 g-2-d} H^{1}\left(\Sigma_{g-1}\right)_{(g-d)}\right] } \\
& \oplus\left[X(g-1, d-1) \otimes H^{*}\left(\coprod_{n-1} S^{1}\right)\right] .
\end{aligned}
$$


The $d_{2}$ differential acts as was determined in Lemma 11 only on the last summand above (and only when $n>1$ ). Thus in "most" positions $(i, j)$ the last factor is killed in homology, with the exception of those $(i, j)$ with $i=0$ or $j=k$ : since the differential maps $(i, j)$ to $(i-1, j-1)$ there are additional cycles when $j=k$ and fewer boundaries when $i=0$. Specifically, the homology of (12) is given by the first term in brackets plus the contributions:

$$
\begin{array}{ll}
(i=0) & \bigoplus_{p=1}^{d} \Lambda^{2 g-2-d+p} H^{1}\left(\Sigma_{g-1}\right) \otimes H^{1}\left(\coprod_{n-1} S^{1}\right) \\
(j=k) & \bigoplus_{p=1}^{d} \Lambda^{2 g-2-d+p} H^{1}\left(\Sigma_{g-1}\right) \otimes H^{0}\left(\coprod_{n-1} S^{1}\right) \otimes U^{p-1} .
\end{array}
$$

where $d=g-1-k$.

Proof of Theorem 1 The results of the preceding sections show that for given $k$ with $0<k \leq g-1$

$$
H F^{+}\left(M\left(t_{\sigma}^{n}\right), \mathfrak{s}\right) \cong H_{*}(C\{i<0 \text { and } j \geq k\})
$$

where the right-hand side refers to the homology of the indicated quotient complex of $C F K^{\infty}(Y, \tilde{K})$ (in the torsion $\operatorname{spin}^{c}$ structure on $\left.Y=M_{n} \#(2 g-2) S^{1} \times S^{2}\right)$. The latter has been shown to be isomorphic as a relatively graded $\mathbb{Z}$-module to the sum of the first bracketed term in (12) and the two expressions (13) and (14).

To understand those two expressions, recall that the summand $\Lambda^{2 g-q} H^{1}\left(\Sigma_{g}\right) \otimes U^{j}$ of $X(g, d)$ is supported in grading $g-q-2 j$. From this it follows (recall that the factors in (13) and (14) arise from $X(g-1, d-1)$ as in the second term of (12)) that

$$
\begin{aligned}
\text { (13) } \oplus \text { (14) } & \cong(n-1) \bigoplus_{p=1}^{d} \Lambda_{(g-d+p)}^{2 g-2-d+p} H^{1}\left(\Sigma_{g-1}\right) \oplus \Lambda_{(g-d-p+1)}^{2 g-2-d+p} H^{1}\left(\Sigma_{g-1}\right) \\
& \cong \bigoplus_{p=1}^{d} \Lambda_{(g-d-p+1)}^{2 g-2-d+p} H^{1}\left(\Sigma_{g-1}\right) \otimes H_{*}\left(\coprod_{n-1} S^{2 p-1}\right) .
\end{aligned}
$$

Adding this to the first line of (12) gives (11), when $k$ is positive. For negative $k$, the result follows from the conjugation invariance of $\mathrm{HF}^{+}$(see [4]).

3.4. The Case of Left-Handed Twists. The procedure for calculation of $\mathrm{HF}^{+}\left(M\left(t_{\sigma}^{-n}\right)\right)$, $n>0$, is very similar to the positive-twist case. We outline here the main differences.

The surgery diagram for $M\left(t_{\sigma}^{-n}\right)$ is identical to that for $M\left(t_{\sigma}^{n}\right)$ with the exception that -1-surgery curves corresponding to the Dehn twists are replaced by +1 -curves (c.f. Figure 11). Thus, $M\left(t_{\sigma}^{-n}\right)$ is obtained by 0 -surgery on the knot $\tilde{K}=\#(g-$ 1) $B(0,0) \# K$ as before, where $K \subset M_{-n}$ is as in Figure 2(a) with signs changed, or as in Figure 2(b) with positive twists replaced by negative ones. Since $M_{-n}$ is just $M_{n}$ with the orientation reversed, we have (using the behavior of $\widehat{H F}$ under orientation reversal) $\widehat{H F}\left(M_{-n} ; \mathfrak{s}_{0}\right)=\mathbb{Z}_{(0)}^{n+1} \oplus \mathbb{Z}_{(-1)}^{n+1}$. 
To obtain the knot Floer homology $\widehat{H F K}\left(M_{-n}, K\right)$, we proceed as before: the result corresponding to Lemma 8 is

$$
\widehat{\operatorname{HFK}}\left(M_{-1}, K ; j\right)= \begin{cases}\mathbb{Z}_{(1)} & j=1 \\ \mathbb{Z}_{(-1)} \oplus \mathbb{Z}_{(0)}^{3} & j=0 \\ \mathbb{Z}_{(-1)} & j=-1 \\ 0 & \text { otherwise }\end{cases}
$$

This is proved in just the same way as Lemma 8 with some surgery coefficients having opposite sign (see [2]).

In general, the analogue of Proposition 9 is:

$$
\widehat{H F K}\left(M_{-n}, K ; j\right)= \begin{cases}\mathbb{Z}_{(1)} & j=1 \\ \mathbb{Z}_{(-1)}^{n} \oplus \mathbb{Z}_{(0)}^{n+2} & j=0 \\ \mathbb{Z}_{(-1)} & j=-1 \\ 0 & \text { otherwise }\end{cases}
$$

and is proved in an analogous manner. Put another way,

$$
\widehat{H F K}\left(M_{-n}, K ; j\right) \cong \Lambda^{*} H^{1}\left(\Sigma_{1}\right) \oplus H^{*}\left(\coprod_{n} S^{1}\right)[-1],
$$

where "[-1]" indicates that the grading on the corresponding factor has been shifted down by 1 (the grading on the exterior algebra, as usual, is centered). Note that the differential $d_{1}$ in the spectral sequence calculating $\widehat{H F}\left(M_{-n}\right)$ from the above consists of an injection $\mathbb{Z}_{(1)} \rightarrow \mathbb{Z}_{(0)}^{n+2}$, which we may identify with contraction by a generator in $H_{1}\left(\Sigma_{1}\right)$.

It follows just as in the previous work that

$$
\widehat{H F K}(Y, K) \cong \Lambda^{*} H^{1}\left(\Sigma_{g}\right) \oplus\left(\Lambda^{*} H^{1}\left(\Sigma_{g-1}\right) \otimes H^{*}\left(\coprod_{n} S^{1}\right)[-1]\right),
$$

and in the spectral sequence calculating $H F^{+}\left(M\left(t_{\sigma}^{-n}\right)\right)$,

$$
E_{1}=X(g, d) \oplus\left(X(g-1, d-1) \otimes H^{*}\left(\coprod_{n} S^{1}\right)[-1]\right) .
$$

The $d_{1}$ differential in this case again acts only on the first factor above, and is given by equation (9). However, under the splitting $E_{+} \oplus E_{-}$of the first factor (c.f. the remarks after equation (8) ), here $d_{1}$ is trivial on $E_{-}$and acts nontrivially only on $E_{+}$. The homology of $X(g, d)$ with respect to this differential was also calculated in [2], and that result gives

$$
\begin{aligned}
E_{2}= & X(g-1, d-1) \otimes H^{*}\left(S^{1}\right)[-1] \oplus \Lambda^{2 g-2-d} H^{1}\left(\Sigma_{g-1}\right)_{(g-d)} \\
& \oplus\left(X(g-1, d-1) \otimes H^{*}\left(\coprod_{n} S^{1}\right)[-1]\right) \\
\cong & X(g-1, d-1) \otimes H^{*}\left(S^{1} \sqcup S^{1}\right)[-1] \oplus \Lambda^{2 g-2-d} H^{1}\left(\Sigma_{g-1}\right)_{(g-d)} \\
& \oplus\left(X(g-1, d-1) \otimes H^{*}\left(\coprod_{n-1} S^{1}\right)[-1]\right) .
\end{aligned}
$$


An argument as in the positive case identifies the $d_{2}$ differential as being nontrivial only on the second factor above, mapping

$d_{2}: \Lambda^{k} H^{1}\left(\Sigma_{g-1}\right) \otimes H^{0}\left(\coprod_{n-1} S^{1}\right)[-1] \otimes U^{j} \longrightarrow \Lambda^{k} H^{1}\left(\Sigma_{g-1}\right) \otimes H^{1}\left(\coprod_{n-1} S^{1}\right)[-1] \otimes U^{j+1}$

isomorphically. The resulting homology is given again by the sum of (13) and (14), this time with a grading shift of -1 . This proves Theorem 1 in the case $n<0$.

\section{REFERENCES}

[1] E. Eftekhary, Floer cohomology of certain pseudo-Anosov maps, math.SG/0205029

[2] S. Jabuka and T. Mark, Heegaard Floer homology of certain mapping tori, Alg. Geom. Topol., 6 (2004) 685-719.

[3] I. G. Macdonald, Symmetric products of an algebraic curve, Topology 1 (1962), 319-343.

[4] P. Ozsváth and Z. Szabó, Holomorphic disks and topological invariants for closed 3-manifolds, to appear in Annals of Math. math.SG/0101206

[5] P. Ozsváth and Z. Szabó, Holomorphic disks and three-manifold invariants: properties and applications, to appear in Annals of Math. math.SG/0105202

[6] P. Ozsváth and Z. Szabó, Holomorphic triangles and invariants for smooth four-manifolds, math.SG/0110169

[7] P. Ozsváth and Z. Szabó, Absolutely graded Floer homologies and intersection forms for four-manifolds with boundary, Adv. Math. 173 (2003), no. 2, 179-261.

[8] P. Ozsváth and Z. Szabó, Holomorphic disks and knot invariants, Adv. Math. 186 (2004), no. $1,58-116$.

[9] P. Ozsváth and Z. Szabó, Heegaard Floer homology and alternating knots, Geom. Topol. 7 (2003) 225-254

[10] P. Seidel, The symplectic Floer homology of a Dehn twist, Math. Res. Lett. 3 (1996), no. 6, 829-834. 